\title{
FAMUSAMM: An Algorithm for Rapid Evaluation of Electrostatic Interactions in Molecular Dynamics Simulations
}

\author{
M. EICHINGER, H. GRUBMÜLLER, H. HELLER, P. TAVAN \\ Institut für Medizinische Optik, Theoretische Biophysik, Ludwig Maximilians Universität München, \\ Theresienstr. 37, D-80333 München, Germany
}

Received 24 October 1996; accepted 22 April 1997

\begin{abstract}
Within molecular dynamics simulations of protein-solvent systems the exact evaluation of long-range Coulomb interactions is computationally demanding and becomes prohibitive for large systems. Conventional truncation methods circumvent that computational problem, but are hampered by serious artifacts concerning structure and dynamics of the simulated systems. To avoid these artifacts we have developed an efficient and yet sufficiently accurate approximation scheme which combines the structureadapted multipole method (SAMM) [C. Niedermeier and P. Tavan, J. Chem. Phys., 101, 734 (1994)] with a multiple-time-step method. The computational effort for MD simulations required within our fast multiple-time-step structureadapted multipole method (FAMUSAMM) scales linearly with the number of particles. For a system with 36,000 atoms we achieve a computational speed-up by a factor of 60 as compared with the exact evaluation of the Coulomb forces. Extended test simulations show that the applied approximations do not seriously affect structural or dynamical properties of the simulated systems. (C) 1997 John Wiley \& Sons, Inc. J Comput Chem 18: 1729-1749, 1997
\end{abstract}

Keywords: molecular dynamics; protein dynamics; fast electrostatics computation; fast multipole method; multiple-time-step method 


\section{Introduction}

n many cases, dynamical processes contribute essentially to protein function (e.g., in ligand binding or in enzymatic reactions). Representing a microscopic description of protein dynamics in the framework of classical mechanics, molecular dynamics (MD) simulations ${ }^{1-3}$ can serve as a tool to interpret experimental data and to provide predictions on hitherto unobserved processes.

MD simulations of protein dynamics pose a computational challenge, because suitable simulation systems have to include the native environment (e.g., water and lipid molecules) of these biological macromolecules ${ }^{4-7}$ and, therefore, typically have to comprise several tens of thousands of atoms. Furthermore, they have to use femtosecond integration time steps to enable smooth descriptions of the fastest degrees of freedom. Simulations of such systems are currently limited to nanoseconds even if the most powerful supercomputers are employed. Although there are biochemically important processes that actually take place on a nanosecond time scale, ${ }^{8}$ and have been successfully described by MD simulations, ${ }^{9,10}$ most biochemical processes occur on time scales well above nanoseconds and, thus, are inaccessible to conventional MD methods.

In the MD approach the classical Newtonian equations are solved numerically to obtain the motion of all atoms. ${ }^{11-13}$ For each numerical integration step, the Coulomb sum:

$$
U \propto \sum_{i}^{N} \sum_{j<i} \frac{q_{i} q_{j}}{\left|\mathbf{r}_{i}-\mathbf{r}_{j}\right|}
$$

between all pairs of atoms $(i, j)$ with partial charges $q_{i}$ and positions $\mathbf{r}_{i}$ has to be evaluated. In common force fields for protein and solvent molecules most atoms carry partial charges; therefore, the evaluation of $U$ dominates the computational effort in MD simulations as it scales quadratically with the number $N$ of charged particles.

In truncation methods, ${ }^{14}$ which simply neglect long range electrostatic interactions beyond a cutoff distance of typically 8-10 $\AA$, the computation of $U$ scales with $N$ instead of $N^{2}$ and, consequently, such methods are widely used in MD simulations. However, the truncation of electrostatic interactions leads to serious errors in forces, energies, and other observables. These errors are generally too large to be acceptable for descriptions of protein dynamics. ${ }^{15-18}$ For instance, using a cutoff distance of $10 \AA$ the relative errors in the computation of electrostatic forces are of the order of $10 \% .{ }^{19}$ One of the major artifacts concerning the description of protein dynamics, that is entailed by these sizable errors, is a suppression of dynamical correlations between slowly fluctuating protein modes, as these are mainly coupled by the neglected long-range Coulomb forces. ${ }^{16,18}$ Thus, truncation of electrostatics trades a description of protein dynamics which is computationally prohibitive but adequate in the sense of basic physics for a description which is computationally manageable but physically inadequate.*

As a result of the above considerations, one of the major tasks which has to be tackled in the strive for improved MD methods is the design of approximate algorithms for computationally efficient and sufficiently accurate evaluation of longrange Coulomb interactions and integration of the Newtonian equations.

However, any attempt to solve the problems just stated is confronted by two difficulties: First, requiring efficiency and accuracy on equal footings typically represents a contradiction, because approximate algorithmic schemes tend to gain computational efficiency at the cost of accuracy; that is, contradiction already has shown up in the above discussion of truncation methods and has to be taken care of in the design of any new approximation scheme. Second, it is much simpler to estimate the computational complexity of an approximate MD algorithm, because this is just a matter of counting floating point operations, than to judge its accuracy. In fact, as discussed in Refs. 16 and 20 , the latter task is highly nontrivial due to the complicated, nonlinear, and chaotic dynamics of macromolecular systems and requires careful comparisons of a variety of statistical observables on structural and dynamical properties that have to be extracted from extensive sample simulations. For instance, the particular artifact of the truncation method noted above, that is, the suppression of dynamical correlations, became apparent only after the progress of computer technology had enabled simulations of sufficiently long duration. To the extent that quality assessments of approximate MD algorithms will become necessary in the

\footnotetext{
*Also the computation of van der Waals forces required in MD simulations scales with $N^{2}$; however, as these forces decay much more rapidly with increasing interaction distance than the Coulomb forces, they can safely be truncated at about $10 \AA$ and, therefore, are computationally inexpensive.
} 
present study, we will adopt the problem-adapted approach developed in Refs. 16 and 20 and will employ the procedures and some of the statistical observables that have been suggested there as useful and suitable tools to judge algorithmic accuracy (see subsequent text).

Up to now various approaches have been suggested that aim at a more efficient approximate treatment of the long-range electrostatic interactions in MD simulations. These approaches may be classified into two categories: multipole ${ }^{18,19,21-26}$ and multiple-time-step methods. ${ }^{16,27-33}$

Multipole methods approximate the long-range forces originating from a group of point charges by truncated multipole expansions of their electrostatic potential. Using a hierarchy of grids for subdivision of space, nested on multiple scales, and a corresponding hierarchical organization of charge groups and multipole expansions, ${ }^{22}$ a computational complexity of $O(N \log N)$ is achieved. By additionally using a hierarchy of local Taylor expansions for the evaluation of the electrostatic forces acting on particles, Greengard and Rokhlin have constructed the so-called fast multipole method (FMM) that even scales with $O(N)$ for large systems. ${ }^{23-25}$ Several implementations of this method suited for MD simulations have been reported. ${ }^{34-36}$

For MD simulations of biomolecules, the FMMtype grouping of charges, defined by a fixed and regular subdivision of space, requires multipole expansions of rather high order to achieve sufficient numerical accuracy. ${ }^{24}$ If, instead, charge grouping is adapted to structural and dynamical properties of the simulated biomolecules, multipole expansions can be truncated at low orders without large sacrifices of accuracy. ${ }^{18,19,26}$ Accordingly, such a structure-adapted multipole method (SAMM) provides further substantial speed-up for MD simulations.

Multiple-time-step methods are based on the observation that forces between distant atoms generally exhibit slower fluctuations than forces between close atoms. Therefore, without significant loss of accuracy, the more slowly fluctuating forces can be treated with longer integration step sizes. The required separation of forces can be implemented, for example, by grouping atom pairs into distance classes. Simple multiple-time-step methods define only two distance classes, an inner and an outer one, ${ }^{27}$ whereas more advanced methods employ a hierarchy of such classes. ${ }^{16,28-33}$

As one can expect from the general considerations just presented, the enhanced efficiency of multipole and multiple-time-step methods is ob- tained at the cost of various kinds of algorithmic artifacts. A typical example is the artificial introduction of "algorithmic noise," which arises from discontinuous and discretized representations of continuous and smooth functions and processes. Such noise can effectively act like an uncontrolled stochastic force on the atomic motion, and can turn the simulated dynamics into a Langevin-type dynamics with the unwelcome property that the algorithmic noise is not properly balanced by a corresponding friction term and, therefore, constantly adds heat to the simulated system. By thorough studies of sample simulations these artifacts have been shown to be negligible in the case of the SAMM method, ${ }^{18,19,26}$ and to be optimally small in the case of a particular multiple-time-step procedure, the so-called DC-1d extrapolation scheme, which has been suggested and carefully compared with other methods in Ref. 16.

From a general point of view, multipole methods and multiple-time-step methods exploit different regularities of Coulomb interactions as to obtain enhanced computational efficiency: the former make use of regularities in space, whereas the latter exploit regularities in time. This complementarity led us to propose ${ }^{16}$ that a combination of both concepts should be able to render additional speed-ups. In view of the proven superiority of the SAMM method as compared to fixed-grid FMM procedures, on the one hand, and of the DC-1d scheme as compared with other multiple-time-step schemes, on the other hand, these two methods appear to be natural candidates for the envisaged combination. It is the purpose of the present work to show how a suitable combination of these methods can be achieved and to demonstrate that the resulting fast multiple-time-step structure-adapted multipole method (FAMUSAMM) actually preserves and combines the advantages of the parent methods with respect to enhanced computational efficiency and to the lack of substantial algorithmic artifacts. In particular, we will demonstrate, by comparison of a combination of SAMM with other multiple-time-step schemes, that the DC-1d procedure is actually the method of choice.

Windemuth ${ }^{37}$ has recently presented a first step toward a combination of multipole and multipletime-step methods; using a simple variant of a multiple-time-step method with only two distance classes and a conventional FMM algorithm he achieved considerable speed-ups. Unfortunately, a quality assessment is lacking in Windemuth's study. Zhou and Berne ${ }^{38}$ reported a more advanced combination of the multiple-time-step al- 
gorithm RESPA ${ }^{31}$ with a slightly modified fast multipole method using a rectangular subdivision of the simulation volume. Here, quality assessments are based on short MD simulations in the picosecond range. For MD simulations with periodic boundaries $^{39-41}$ a fast method, based on a combination of RESPA and the smooth particle mesh Ewald method, ${ }^{42}$ has been described. ${ }^{43}$ The latter method is particularly useful for simulations of crystalline structures (e.g., protein crystals). Also, for the simulation of noncrystalline systems, like in lipid-membrane-soluted proteins, ${ }^{4}$ periodic boundaries have been used as an approximation due to the availability of efficient algorithms. In the latter case, however, it is not clear a priori whether that approximation is justified (for a discussion of possible artifacts see, e.g., Refs. 44-47). In our study, we develop an efficient algorithm to speed up simulations of nonperiodic systems, in which, typically, the environment is effectively included through the use of appropriate boundary forces (see, e.g., Refs. 44 and 48). We provide a careful analysis of possible algorithmic artifacts and their influence on structural and dynamical properties of a test system.

We intend to achieve high efficiency by combining SAMM and a multiple-time-step scheme. To that aim, we first outline the basic features of the parent methods and, subsequently, present our new algorithm, FAMUSAMM. As will be explained in the following sections, in our multipole method, charge grouping will be based on the specific structural features of macromolecules rather than on fixed subdivisions of space as used in the methods cited previously. In particular, our combination of SAMM with a multiple-time-step method is expected to be more efficient and less memory consuming, because we intend to apply the multiple-time-step scheme to local Taylor expansions of the electrostatic potential rather than to Coulomb forces acting on individual atoms. Having defined suitable methods to evaluate FAMUSAMM's accuracy, we present the results of our accuracy assessment. After documenting the enhanced computational performance of our method, we discuss and summarize our results.

\section{FAMUSAMM Algorithm}

As indicated previously, we intend to combine two well-established methods for rapid evaluation of long-range Coulomb interactions into a new and improved algorithm. To enable an understanding of the computational strategy to be implemented by our combination, we first sketch the basic concepts of its parent methods, SAMM and the multiple-time-step method DC-1d.

\section{STRUCTURE-ADAPTED MULTIPOLE METHOD}

The SAMM method ${ }^{18,19,26}$ exploits structural features of biomolecules to define a hierarchical grouping of partially charged atoms into local charge distributions, whose multipoles are subsequently used for evaluation of electrostatic interactions at large distances.

Figure 1 illustrates the hierarchy of charge distributions ("objects") obtained by the SAMM-type grouping (left) and the electrostatic representations chosen for the resulting objects (right). Both the definition of the objects and the choice of their electrostatic representations rest on the observation that covalently bound atoms within biomolecules intrinsically form dynamically stable local groups, which either carry integer elementary charges or are uncharged, but dipolar. In case the latter dipoles are small, the local groups are con-

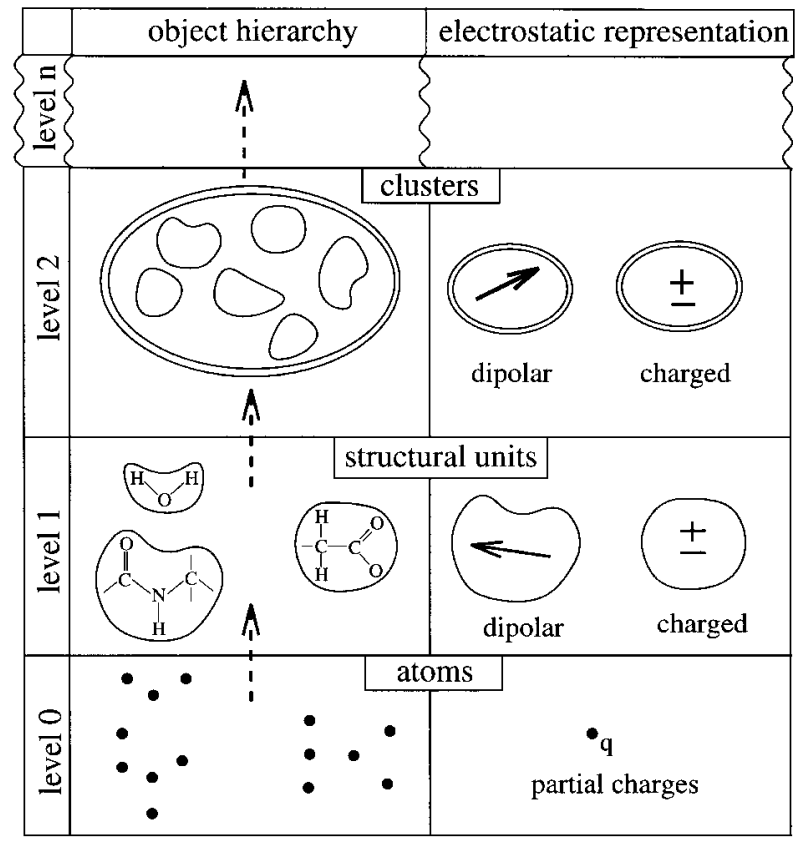

FIGURE 1. Structure-adapted hierarchical description of biological macromolecules. Filled circles represent atoms, structural units are surrounded by a single-line border, and clusters are surrounded by a double-line border. 
sidered nonpolar and their electrostatics may be neglected. ${ }^{18,19}$

This observation suggests that, at larger distances, the lowest multipole moments of local groups can provide an accurate description of the electrostatics within biomolecules. Thus, charged local groups should be described by monopoles and dipolar groups by dipoles for the description of their electrostatic interactions at large distances. In the SAMM method that concept represents the guideline for the definition of the object hierarchy illustrated in Figure 1. As shown in the figure, at the bottom of the object hierarchy (level 0), no local groups are formed; instead, individual atoms (filled circles) are considered as the basic objects and are electrostatically represented by their partial charges. ${ }^{\dagger}$ At small distances (i.e., at distances smaller than a predefined distance $D_{1}$ ), their interactions are evaluated by means of the Coulomb sum [eq. (1)]. For a description of electrostatic interactions at larger distances covalently bound atoms are grouped into structural units which are compact, typically include three to ten atoms, and form the first level of the object hierarchy (level 1). According to their respective electrostatic properties, SAMM distinguishes three types of structural units: (1) "neutral units" consist of uncharged atoms only, and they need not be considered and are therefore omitted in Figure 1; (2) "dipolar units" (e.g., water molecules or peptide groups of a protein backbone) are composed of partially charged atoms, exhibit a vanishing net charge, and are represented by a dipole (arrow); and (3) "charged units" (e.g., charged side groups of amino acids) carry an integer net charge and are represented by a monopole. At all higher levels (level 2 and further up) pertaining to interactions at still larger distances (i.e., at distances larger than a second, predefined interaction distance $D_{2}$ ) objects of the preceding lower level are grouped into clusters by means of self-organizing and adaptive vector quantization techniques. ${ }^{49}$ For a predefined number of clusters these techniques manage to minimize cluster extension and, thereby, also the approximation error caused by the truncation of the multipole expansions. ${ }^{18,19}$ Depending on their total charge the clusters are represented either by

\footnotetext{
${ }^{\dagger}$ In a heteronuclear molecule each atom carries a partial charge; however, usual parameterizations of protein force fields are chosen such that small partial charges are neglected while the sum of partial charges is locally kept at integer values.
}

dipoles or by monopoles. We use $D_{1}=10 \AA$ and $D_{2}=16 \AA$ for the interaction distances associated with the hierarchy levels; that choice has been demonstrated to guarantee highly accurate descriptions. ${ }^{19}$

Computationally, the hierarchical multipole expansions of SAMM are defined and evaluated by a bottom-up scheme: The monopoles and dipoles of the structural units at level 1 are calculated from the individual charges of their enclosed atoms; at the second level, the monopoles and dipoles of the clusters are obtained from the respective multipole moments of their embodied structural units and so forth (for further details see Ref. 18).

Note that conformational changes in the protein or diffusive motions of solvent molecules would lead to an increase of cluster sizes in the course of an MD simulation, if the grouping of structural units into clusters were to be chosen as fixed. As a result, the accuracy of the electrostatic representation by the truncated multipole expansions would decrease. To maintain the accuracy of description SAMM periodically regroups the structural units into new clusters applying the adaptive techniques previously mentioned. In principle, such regrouping represents a discontinuity of description and, thus, is a source of algorithmic noise. However, the chosen adaptive reclustering procedure assures that the redistribution of structural units among clusters is kept minimal, and, therefore, also minimizes algorithmic noise. ${ }^{18,19}$

In addition to the diligent choice of structural units and of optimally compact clusters the SAMM method provides a further means to reduce the approximation error connected with the truncated multipole expansions, i.e., the choice of optimal reference points of the multipole expansions. This strategy is based on the fact that the first nonvanishing moment of a multipole expansion, in contrast to the higher moments, is independent of the reference point. As SAMM considers only the first nonvanishing moment, the truncation error can be made optimally small by an adequate choice of the reference point. ${ }^{18,19}$

Whereas the original version of SAMM ${ }^{19}$ comprises solely a hierarchical multipole scheme, its enhanced version ${ }^{26}$ also utilizes FMM strategies. ${ }^{23,24}$ Here, local Taylor expansions of the elec-

\footnotetext{
${ }^{\ddagger}$ In contrast to the original SAMM version, we now also combine monopoles of opposite sign into dipolar objects at higher levels, if the total charge of these objects vanishes. This procedure allows representation of the electrostatics of neighboring ion pairs at large distances as dipoles.
} 
trostatic potential originating from distant charge distributions and their associated multipole moments are used to speed up the calculation of forces acting on partial charges in a given neighborhood. A careful analysis of sample simulations has shown that the local Taylor expansion may be safely truncated at second order, if the SAMMspecific distance classes are applied, that is, if forces originating from objects more distant than $D_{1}$ are considered. ${ }^{18,26}$ Thus, the fast version of SAMM approximates the corresponding electrostatic potential $\Phi(\mathbf{R})$ at position $\mathbf{R}=\mathbf{R}_{o}+\mathbf{r}$, within the volume of a structural unit or cluster in terms of its value and derivatives at the optimized reference point, $\mathbf{R}_{o}$, of that object by the local Taylor expansion:

$$
\Phi^{L}\left(\mathbf{R}_{o}+\mathbf{r}\right)=\Phi_{o}+\sum_{\alpha=1}^{3} K_{\alpha} r_{\alpha}+\frac{1}{2} \sum_{\alpha, \beta=1}^{3} T_{\alpha \beta} r_{\alpha} r_{\beta}
$$

where the expansion coefficients are defined by:

$$
\begin{gathered}
\Phi_{o} \equiv \Phi\left(\mathbf{R}_{o}\right),\left.\quad K_{\alpha} \equiv \frac{\partial \Phi(\mathbf{R})}{\partial R_{\alpha}}\right|_{\mathbf{R}=\mathbf{R}_{o}} \\
\text { and }\left.T_{\alpha \beta} \equiv \frac{\partial^{2} \Phi(\mathbf{R})}{\partial R_{\alpha} \partial R_{\beta}}\right|_{\mathbf{R}=\mathbf{R}_{o}}
\end{gathered}
$$

In eqs. (2) and (3), Greek indices enumerate Cartesian coordinates.

Figure 2 illustrates the FMM aspect of SAMM for the lowest three hierarchy levels, $H=0,1,2$. As a function of object distance $d$ it is shown how objects at different hierarchy levels contribute (long arrows) to the electrostatic force acting on a selected atom ( $\$$ in Fig. 2). Three corresponding distance classes $\left(d \leq D_{1}, D_{1}<d \leq D_{2}, D_{2}<d\right)$ are separated by vertical dotted lines. Within these distance classes the figure displays those objects that contribute to the force acting on objects of the respective hierarchy level. These objects, which contain the selected atom and which we correspondingly call selected objects, are shown within the leftmost column. For the evaluation of the total electrostatic force on the selected atom the following algorithmic steps have to be performed in a top-down fashion:

Step 1: At the highest hierarchy level $(H=2$ in Fig. 2) the electrostatic potential generated by the multipole moments of all those clusters, which are separated from the se-

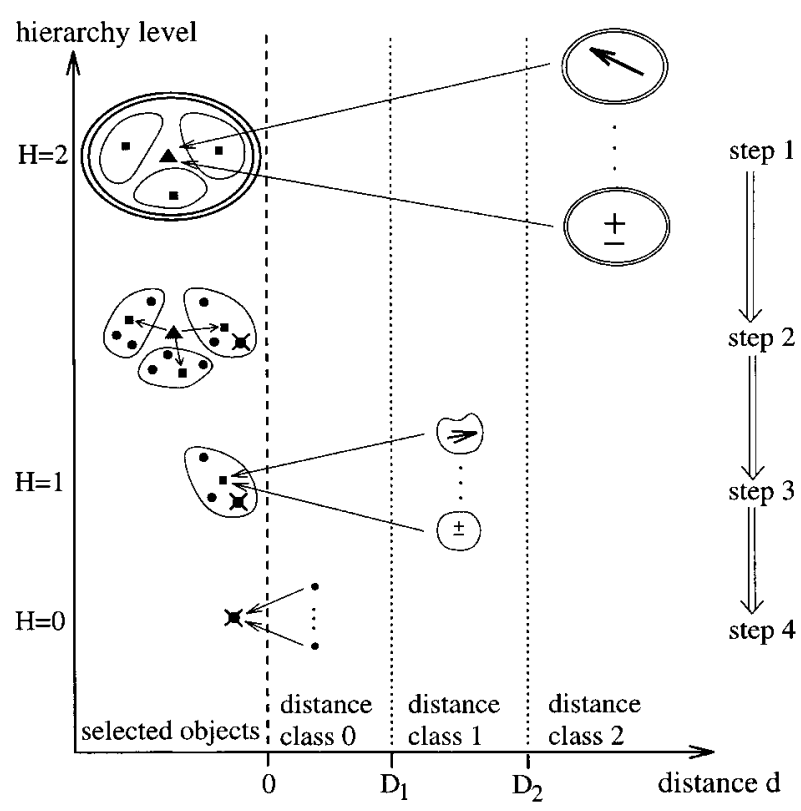

FIGURE 2. Algorithmic steps connected with the local Taylor expansion in the force calculation of the fast version of SAMM; the contributions to the force on a selected atom ( $)$ originating from objects located within various distance classes and associated to corresponding hierarchy levels $H$ are indicated by long arrows; the reference points of the local Taylor expansions are denoted by triangles $(\boldsymbol{\Lambda})$ for clusters $(H=2)$ and by squares ( $\square)$ for structural units $(H=1)$; short arrows starting at a reference point indicate the "inheritance of local Taylor expansions." For explanation see text.

lected cluster by more than the second interaction distance $D_{2}$, is approximated within the selected cluster by a local Taylor expansion of the electrostatic potential using the reference point ( $\Delta$ in Fig. 2).

Step 2: Now the local Taylor expansion of the selected cluster is transformed (short arrows) to local Taylor expansions of its embodied structural units. This procedure is called "inheritation," and is the key to the high efficiency of FMM schemes. The transformation is accomplished by shifting the reference point from the center of the selected cluster ( $\Delta$ in Fig. 2) to the centers ( in Fig. 2) of its embodied structural units. ${ }^{18,26}$ The resulting Taylor expansions of the structural units now describe the same electrostatic potential as their "parent" local Taylor expansion.

Step 3: At the next lower hierarchy level $(H=$ 1 in Fig. 2) we consider additional electrostatic interactions; that is, those of the se- 
lected structural unit with structural units in a distance range between $D_{1}$ and $D_{2}$. Their contribution to the electrostatic potential within the selected structural unit is again approximated by a local Taylor expansion and is added to the local Taylor expansion inherited from the higher levels. The resulting local Taylor expansion approximates the electrostatic potential generated by all atoms more distant than $D_{1}$.

Step 4: At the lowest hierarchy level, $H=0$, the local Taylor expansion of the selected structural unit is evaluated at the position of the selected atom. Interactions with atoms at distances smaller than $D_{1}$ are calculated directly from the corresponding Coulomb sum [eq. (1)] and are added.

The above presentation of the FMM strategy utilized by SAMM completes the outline of that algorithm. We have seen how SAMM takes advantage of structural properties of protein-solvent systems and how this method exploits distancedependent spatial regularities of the corresponding electrostatic potential to construct a computationally fast, yet accurate approximation scheme.

\section{MULTIPLE-TIME-STEP METHODS}

Another class of approximation schemes, the multiple-time-step methods, ${ }^{16,28,29,32,33}$ exploit complementary properties of protein electrostatics for the same purpose; they take advantage of temporal regularities. The so-called distance class methods, in particular, are based on the observation that forces originating from distant atoms fluctuate more slowly than forces from atoms nearby (see Fig. 3). The slowly fluctuating forces may be evaluated less frequently than the fast ones and may be extrapolated at the time steps in between. Such extrapolation is required as the numerical integration of the dynamical equations needs all forces at every integration time step $\tau=0,1,2, \ldots$, which discretizes the simulation time $t=\tau \Delta t$, where $\Delta t$ is the integration time step size. ${ }^{\S}$

The left part of Figure 3 illustrates how distance classes $j$ can be defined by a set of increasing radii $R_{j+1}$. The set of atoms $\{m\}$ at positions $\mathbf{r}_{m}$, satisfying $R_{j} \leq\left|\mathbf{r}_{l}-\mathbf{r}_{m}\right|<R_{j+1}$, makes up the distance

\footnotetext{
${ }^{\S}$ In MD simulations, $\Delta t$ is usually set to $1 \mathrm{fs}$, which is short enough to smoothly describe the fastest degrees of freedom in protein simulations.
}

class $j$ of particle $l$ at position $\mathbf{r}_{l}$. As is also indicated in Figure 3 (right part), for each particle $l$ the sum of forces $\mathbf{F}^{(j)}$ arising from particles in distance class $j$ is calculated explicitly every $n_{j}$-th integration time step (filled squares). Each of these time steps is called a macrointegration step. A common choice ${ }^{16}$ is $n_{j}=2^{j}$. Correspondingly, the $2^{j}-1$ elementary time steps within the cycle of a macrointegration step are called microintegration steps. As illustrated in Figure 3, at each step, $\mathbf{F}^{(j)}$ is estimated from two forces calculated at previous macrointegration steps by:

$$
\mathbf{F}_{n_{j} k+i}^{(j)}=a_{i}^{(j)} \mathbf{F}_{n_{j} k}^{(j)}+b_{i}^{(j)} \mathbf{F}_{n_{j}(k-1)}^{(j)}
$$

using appropriate extrapolation coefficients, $a_{i}^{(j)}$ and $b_{i}^{(j)}$. The lower index of $\mathbf{F}$ denotes the absolute integration time-step number and is expressed in terms of the macrointegration step $k=0,1,2, \ldots$, and the cyclic microintegration step $i=0, \ldots$, $n_{j}-1 ; \mathbf{F}_{n_{j} k}^{(j)}$ and $\mathbf{F}_{n_{j}(k-1)}^{(j)}$ are explicitly calculated at the macrointegration steps $k$ and $k-1$.

The hierarchical extrapolation procedure shown is capable of saving an enormous amount of computer time as it frequently avoids the most timeconsuming step (i.e., the exact evaluation of all interactions). Here computational speed is gained at the cost of an increased demand for memory: for each atom and each distance class two previous forces have to be kept in memory.

Various choices for the extrapolation coefficients $a_{i}^{(j)}$ and $b_{i}^{(j)}$ have been discussed ${ }^{16,33,37}$; both the linear extrapolation (illustrated in Fig. 3) and the so-called DC-1d algorithm have been found to be promising. ${ }^{16}$ Although the linear extrapolation, defined by:

$$
a_{i}^{(j)}=1+\frac{i}{n_{j}} \quad \text { and } \quad b_{i}^{(j)}=1-a_{i}^{(j)}
$$

entails smaller discontinuities for the extrapolated forces than the DC-1d algorithm, it leads to a larger energy transfer into a simulation system by algorithmic noise. ${ }^{16}$ The DC-1d scheme employs the coefficients:

$$
\begin{gathered}
a_{i}^{(j)}=\frac{3 n_{j}^{2}-2 n_{j}+1}{n_{j}\left(n_{j}+1\right)}-3 i \frac{n_{j}-1}{n_{j}\left(n_{j}+1\right)} \\
\text { and } b_{i}^{(j)}=1-a_{i}^{(j)}
\end{gathered}
$$

It is not clear a priori, whether the quoted properties of these extrapolation schemes will pertain if they are combined with the SAMM procedure into 


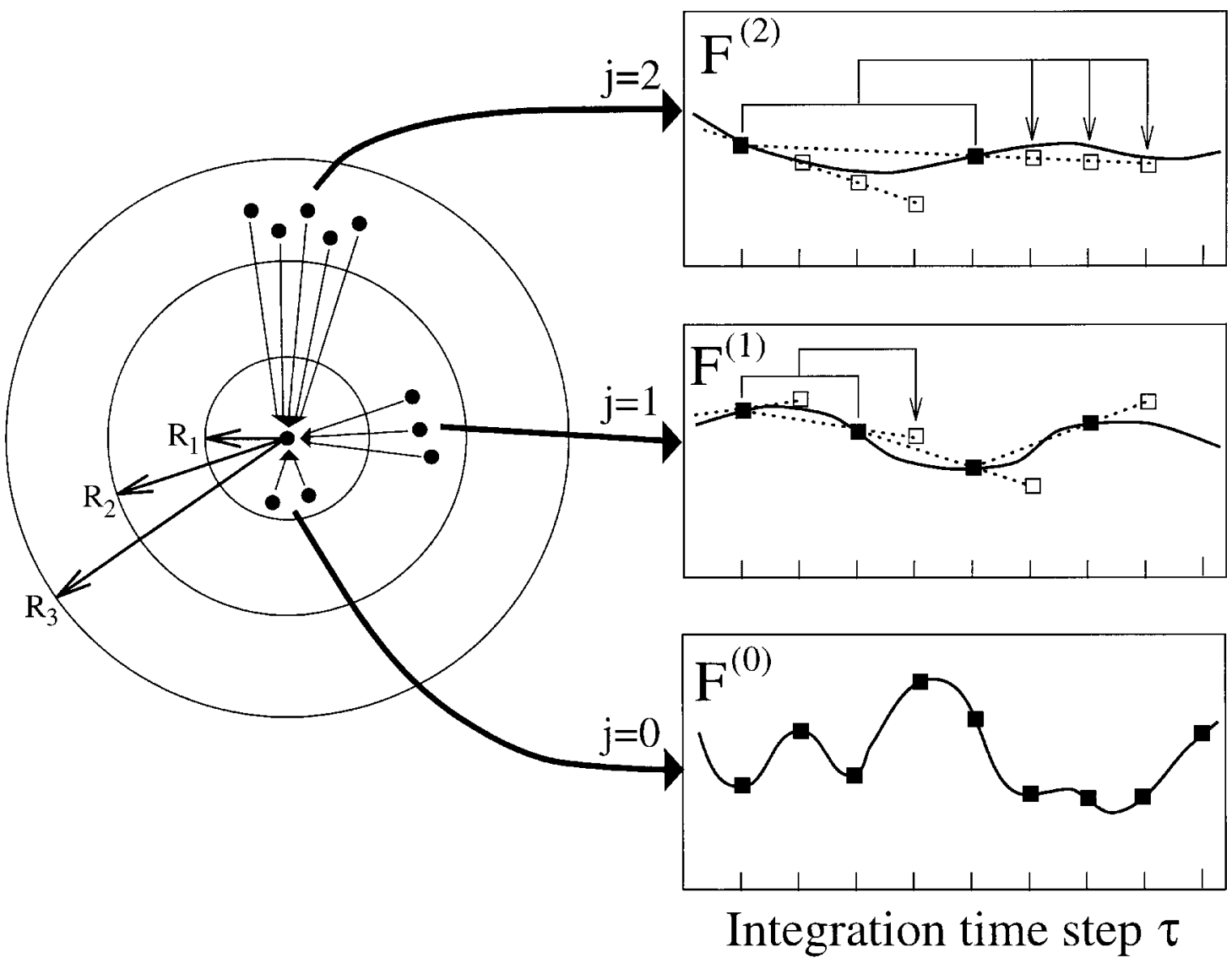

FIGURE 3. Left: Distance classes $j=0,1,2, \ldots$, defined for an atom (central dot) by a set of radii $R_{j+1}$ generating a series of spherical shells. Right: For each distance class the temporal evolution of the total force $\mathbf{F}^{(j)}$ acting on the selected atom originating from all atoms in the respective distance class, along with the exact forces (solid line) and their exact values (filled squares). In addition, linear force extrapolations (dotted lines) and resulting force estimates (empty squares) are depicted.

our new FAMUSAMM scheme, which will be explained in the next section. Therefore, using test simulations we will subsequently check both extrapolation methods for their suitability within our combination method.

\section{COMBINATION OF SAMM WITH A MULTIPLE-TIME-STEP METHOD}

Before starting with the detailed outline of our FAMUSAMM method we annotate some of the important progress concerning its implementation as compared to that of SAMM: The first two SAMM versions ${ }^{18,19,26}$ made use of only two hierarchy levels, which restricted their applicability to medium-sized systems comprising less than about 5000 atoms. To gain substantial speed-ups for larger systems also, we decided upon the design of FAMUSAMM to implement a fast SAMM method with an arbitrary number of hierarchy levels. That number is now determined by the number of atoms in a given simulation system and is chosen automatically in the start-up phase of a simulation. ${ }^{50}$

\section{Basic Concepts}

Our FAMUSAMM algorithm has been designed to further reduce the computational effort for the evaluation of Coulomb forces by exploiting regularities of these forces in space and time. The basis of its development has been the identification of the most time-consuming steps within the fast SAMM algorithm. Only upon such an analysis may one expect that a combination with a multiple-time-step method can render additional speedups. A detailed inquiry of fast SAMM has shown ${ }^{50}$ that the essential time-consuming steps are: (i) the calculation of contributions to the local Taylor ex- 
pansions (see Fig. 2, steps 1 and 3); and (ii) the calculation of the electrostatic forces for the innermost distance class (see Fig. 2, step 4). Therefore, we have focused our efforts on these two steps.

\section{Multiple-Time-Step Extrapolation of Local Taylor Expansions}

We first consider step (i) and remember that the hierarchy of interactions, which is employed for the evaluation of the local Taylor expansions and depicted in Figure 2, is defined by a hierarchy of distances. This hierarchy of distances is intimately related to the particular concept of distance classes underlying the multiple-time-step schemes as discussed above. Therefore, an attempt to combine these methods seems natural.

In multiple-time-step methods, the total force, $\mathbf{F}^{(j)}$, acting on a selected atom originating from distance class $j$ is extrapolated. Because we now want to use a multipole method, we have to identify a corresponding expression for this force. As we have seen in our outline of the SAMM algorithm, the total force originating from outer distance classes and acting on elements contained in a cluster or a structural unit is computed by means of a local Taylor expansion. Such a local Taylor expansion for an object on a given hierarchy level is-except for the topmost level-made up of two components: (a) a contribution inherited from the next higher hierarchy level (see Fig. 2, step 2) comprising the electrostatic interactions with objects in all outer distance classes; and (b) contributions from objects within the same hierarchy level. From the latter one can calculate the desired total force, $\mathbf{F}^{(j)}$, and, therefore, we apply multiple-timestep extrapolations to these contributions. Here, instead of extrapolating the forces directly, we extrapolate the coefficients $\Phi_{0}, K_{\alpha}$, and $T_{\alpha, \beta}$ of the local Taylor expansions, from which the forces derive."

\section{Inner Class Force Extrapolation}

Because of the choice of $10 \AA$ for the interaction distance $D_{1}$ rendering quite a few, typically about 400 , interaction partners for a given partial charge within the innermost distance class $(H=0)$ of SAMM, the second essential time-consuming step

\footnotetext{
"Note that this strategy also saves computer memory, because the local Taylor expansion of a selected object is used to calculate the forces on all particles contained in that object; storage of all these forces would inevitably consume much more memory.
}

(ii) of that method is the calculation of electrostatic forces by the Coulomb sum [eq. (1)]. In fact, this step is equivalent to the one carried out in conventional truncation methods using a cut-off distance $D_{1}$. To reduce that effort by application of a multiple-time-step method, we split this SAMM distance class into two new distance classes, $j=0$ and $j=1$, with the respective radii $R_{1}=5 \AA$ and $R_{2}=10 \AA$. Within these new distance classes we extrapolate forces directly by a multiple-time-step scheme as described in the previous section. Distance classes of similar size have been successfully used previously. ${ }^{16}$

Due to the introduction of these new distance classes, the expensive computation of about 400 interactions per particle is now performed only at every second integration time step, whereas, in distance class $j=0$, only a few interactions, about 50, have to be evaluated at every integration step. Hence, the overall computational effort for the innermost SAMM distance class is reduced nearly by a factor of two.

\section{Interaction List Updates}

Generally, MD methods which are based on distance classes use interaction lists to eliminate the need for time-consuming interaction partner searches at each integration time step. ${ }^{32}$ In FAMUSAMM, that strategy is adopted as follows: For each object at a particular hierarchy level (atom, structural unit, cluster) an associated interaction list points to all objects which belong to the same hierarchy level and meet the distance criterion described previously (cf. Fig. 2). Because distances between atoms, structural units, and clusters vary during an MD simulation, the interaction lists have to be updated from time to time and are likely to change upon these updates. As a result, the forces generated by changing numbers of objects within a given distance class will change discontinuously. The reclustering procedure of SAMM mentioned above causes similar discontinuities. However, the occurrence of such discontinuities disables the application of multiple-time-step extrapolation procedures, because the latter are based on strict smoothness assumptions: Only if the interaction lists and cluster compositions are kept fixed for a sufficiently large ( $\gg 4$, see Initialization subsection) number of integration time steps, multipletime-step extrapolations may be applied. Test simulations have shown that, for our FAMUSAMM interaction lists, update periods covering up to 128 
integration steps can be used; reclustering periods may be even longer and are chosen as integer multiples of the former periods.

\section{Verification of Local Taylor Expansion Smoothness}

As described above, an essential new feature of FAMUSAMM, as compared with previous multiple-time-step schemes, is the extrapolation of local Taylor expansion coefficients for outer distance classes instead of the extrapolation of forces acting on individual atoms. To verify the validity of this approach it remains to be verified whether the smoothness conditions required for multipletime-step extrapolations actually hold for the local Taylor expansion coefficients. Extended test simulations have demonstrated that such is the case, and that local Taylor expansion coefficients exhibit decreasing amplitudes and curvatures with increasing hierarchy level $H$.

\section{Initialization}

A prerequisite for the application of the multiple-time-step extrapolation at a given time step in a simulation is the accessibility to two previously explicitly calculated forces or local Taylor expansion coefficients. As these are absent in the start-up phase of a simulation or, due to the associated discontinuities, cannot be used after an update of the interaction lists, special precautions have to be taken for these periods. ${ }^{32}$ To be specific, the extrapolation of forces or of local coefficients for distance class $j$ requires their values at two previous macrointegration steps, which are separated by $2^{j}-1$ integration steps (see Fig. 3). To provide these values for the initialization periods FAMUSAMM performs exactly $T_{j}=2^{j}$ conventional fast SAMM steps until all necessary forces (for $j=1$ ) or local coefficients (for $j \geq 2$ ) are explicitly calculated. Obviously, the initialization periods, after which multiple-time-step extrapolations can be applied, have different durations, $T_{j}$, for the various distance classes. For the computationally most expensive distant classes, $j=1$ and $j=2$, the initialization lasts only two and four integration steps, respectively. From these numbers it is clear that the efficiency loss due to the initialization phases is negligible, if periods of 64 or more steps are used for the update of the interaction lists.

\section{FAMUSAMM Algorithm}

Figure 4 illustrates the multiple-time-step scheme of FAMUSAMM by which long range Coulomb interactions are evaluated. For a simulation period well after an initialization phase three hierarchy levels, $H$, and a sequence of 20 integration steps are shown. Filled squares indicate explicit fast SAMM force calculations, empty squares indicate extrapolations. The dashed line separates the Coulomb sum interaction classes $(j=0,1)$ from distance classes, which interact by multipole expansions $(j \geq 2)$. For class $j=1$ the extrapolation of forces is used, that is, the respective forces are evaluated only at every second integration step and are extrapolated otherwise; whereas, the electrostatic interactions of partially charged atoms closer than $R_{1}=5 \AA$ (see Fig. 3, $j=0$ ) are calculated at every integration step.

For distance classes $j \geq 2$, which correspond to hierarchy levels $H \geq 1$, the multiple-time-step scheme is applied to the local Taylor expansion coefficients. Here, the local Taylor expansion coefficients of lower hierarchy levels have to be recalculated more often than those of higher hierarchy levels, which, in turn, can be extrapolated more often. As suggested in a previous multiple-timestep method, ${ }^{16}$ explicit recalculation is performed every $2^{j}$-th integration step.

Appendix A summarizes the FAMUSAMM algorithm using a pseudo-code description. The al-

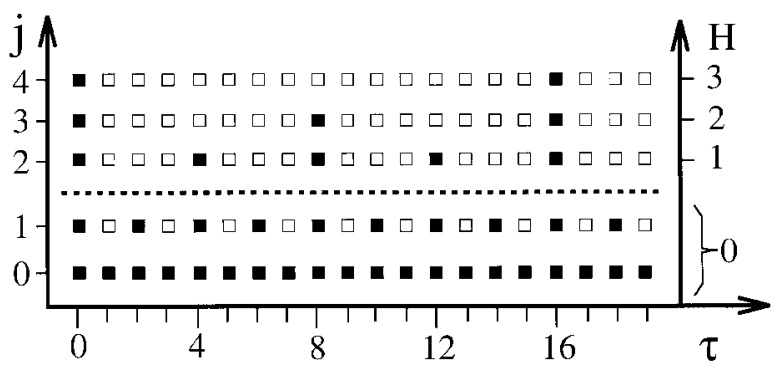

FIGURE 4. Extrapolation scheme of FAMUSAMM. $\tau$ denotes the integration step, $H$ the SAMM hierarchy level, and $j$ the multiple-time-step distance class; filled squares mark the integration steps at which forces originating from objects in distance class $j$ are explicitly calculated, whereas empty squares indicate computationally inexpensive extrapolations. The dashed line separates the regime of force extrapolation in distance class $j=1(H=0)$ from the regime of local Taylor expansion extrapolation in the outer distance classes $j \geq 2(H \geq 1)$; forces originating from partial charges in the innermost distance class $j=0$ are never extrapolated. 
gorithm has been implemented in the MD simulation program EGO_VIII ${ }^{51}$ in a sequential and a parallelized version; the latter is suited for distributed memory parallel computers (e.g., IBM SP2, Cray T3E, Parsytec CC, and workstation clusters under PVM or MPI). Details on the parallelization strategy will be given in a forthcoming publication. $^{52}$

As is apparent from the large number of empty squares depicted in Figure 4, FAMUSAMM replaces quite a few explicit force or local Taylor expansion calculations by extrapolations for the sole purpose of computational efficiency. As noted already in the Introduction, in MD simulations the inaccuracies associated with these FAMUSAMM approximations may effectively act as sources of uncontrolled algorithmic noise. It is the aim of the remaining sections of this article to verify to what extent such numerical artifacts can affect the MD description of structural and dynamical properties of proteins.

\section{Methods of Quality Assessment}

To estimate the quality of the numerous approximations utilized by FAMUSAMM and to measure the gain of computational efficiency we carried out a series of test simulations. We have applied two versions of our method, one employing the linear extrapolation, which we call FAMUSAMM/linear, and one using the DC-1d extrapolation scheme, which we correspondingly denote FAMUSAMM/DC-1d. To enable comparisons with established methods, we have also performed test simulations using a cutoff method, ${ }^{14}$ the fast SAMM algorithm, ${ }^{26}$ and the slow but exact evaluation of the Coulomb sum [eq. (1)], which we use as the reference method.

As discussed in the Introduction, the complicated and chaotic nature of protein dynamics renders quality assessments of approximate MD algorithms a nontrivial task. For example, comparisons of system trajectories or of other atomic details obtained from simulations carried out with different approximation schemes are useless for that purpose, because possible observed deviations merely reflect the chaotic character of these details and thus do not allow derivation of accuracy measures. Instead, following the arguments and suggestions in Refs. 16 and 20, we will apply a set of so-called "relevant" statistical observables for our intended estimates of algorithmic quality. These observables have been selected according to the conditions that they should exhibit regular (i.e., nonchaotic) temporal behavior, and that they should refer to functionally important properties of proteins. ${ }^{16,20}$

\section{MODEL SYSTEMS AND TEST SIMULATIONS}

Our study is divided into two parts. The first part studies effects which are observable within simulation times covering a few picoseconds, and addresses questions like the accuracy of force approximation and the size of the energy drift caused by algorithmic noise. As our sample system, we have chosen the small protein bovine pancreatic trypsin inhibitor (BPTI), which is frequently used as a test system for evaluation of simulation methods. $3,7,53,54$ We embedded BPTI in a water shell of $27-\AA$ radius, comprising a total of 7147 atoms.

The second part serves to estimate algorithmic accuracy with respect to effects on longer time scales. For this purpose, the sample system introduced is prohibitively large as far as the intended reference simulations are concerned, because these require the computationally very expensive evaluation of the Coulomb sum [eq. (1)]. Therefore, we had to choose here a much smaller sample system, namely BPTI in vacuo comprising only 568 atoms. The test simulations from which we have extracted regular statistical observables for our quality estimates covered 1.2 ns each.

Because it is well known that, for realistic protein simulations, the natural environment has to be included, ${ }^{4,6,7,55}$ a note on our choice of an in vacuo test system is necessary: Concerning our long time simulations we do not aim to evaluate the quality of the physical model of the protein, which, here, may be poor. Rather, we want to check to what extent our algorithmic approximations affect the molecular dynamics of our given model. For that purpose, the physical model used here does not necessarily have to be very accurate as long as possible artifacts in realistic applications are likely to show up in our test simulations also.

All test simulations were carried out with the MD program EGO_VIII, ${ }^{51}$ which employs the CHARMM force field. ${ }^{14}$ Nonpolar hydrogen atoms were represented by compound atoms, ${ }^{14}$ and the lengths of chemical bonds involving polar hydrogen atoms were fixed using the SHAKE algorithm. ${ }^{3}$ An integration step size of $1 \mathrm{fs}$ was used. Water molecules were described by the TIP3 model. ${ }^{56}$ 
The interaction list was updated every 64th integration step. For the cutoff simulations, a cutoff distance of $9 \AA$ and a switching function ${ }^{57}$ were chosen. Translations and rotations of the protein were eliminated as described in Refs. 58 and 59. Prior to the test simulations, BPTI in vacuo and the BPTI-water system were equilibrated for 50 ps and 70 ps, respectively, using the reference method. For the long time simulations of BPTI in vacuo the temperature was kept fixed at $300 \mathrm{~K}$ by weakly coupling the system to a heat bath using a coupling constant of $10^{-13} \mathrm{~s}$ as defined in Refs. 60 and 61. In contrast, the short time simulations of the large BPTI-water system were carried out in the microcanonical ensemble to enable an estimate to what extent algorithmic artifacts entail violations of energy conservation.

For the first part of our quality estimates we will compare results of simulations obtained by the supposedly exact reference method with results obtained by SAMM, FAMUSAMM/linear, and FAMUSAMM/DC-1d. Here, comparisons with the cutoff method are superfluous, because related accuracy tests comparing the various versions of SAMM with that method have been published previously. ${ }^{18,19}$ By these tests, the superiority of the SAMM algorithms, as compared with the conventional cutoff procedure, has been convincingly demonstrated. Thus, it solely remains to be verified to what extent our FAMUSAMM approach preserves the advantages of its parent method.

For the second part we have performed 1.2-ns test simulations utilizing all methods under consideration. Here we have included the cutoff approach, because related investigations have not yet been published. Due to the statistical nature of our observables a second 1.2-ns test simulation was necessary for the reference method to enable an estimate of the sizes of statistical fluctuations. Only upon such estimates can algorithmic artifacts be identified and distinguished from inevitable fluctuations of the necessarily statistical observables compared. The initial conditions of the first reference simulation and of the test simulations for the approximate methods were chosen as the atomic positions and velocities obtained after equilibration. For the second reference simulation these initial positions were modified by extremely small random amounts. Note that the chaotic character of protein dynamics ensures complete decorrelation of the two reference simulations within a few picoseconds.

\section{SHOR'T TIME OBSERVABLES}

To measure the numerical accuracy of the force approximation we follow the suggestion in Ref. 19. We consider the root mean square (rms) error of forces:

$$
\eta(t)=\left[\frac{\sum_{i=1}^{N}\left|\mathbf{F}_{i}^{\text {approx }}(t)-\mathbf{F}_{i}^{r e f}(t)\right|^{2}}{\sum_{i=1}^{N}\left|\mathbf{F}_{i}^{r e f}(t)\right|^{2}}\right]^{1 / 2}
$$

where $\mathbf{F}_{i}^{\text {approx }}(t)$ and $\mathbf{F}_{i}^{\text {ref }}(t)$ denote the approximated and the exact Coulomb force, respectively, acting on atom $i$ at time $t$, and where the forces are calculated using the trajectory of the reference simulation. As an accuracy measure we take the mean $\langle\eta\rangle$ of the rms error $\eta(t)$ for the simulated trajectory. The rms error of FAMUSAMM, $\eta(t)$, will fluctuate in the course of a simulation, because, as is apparent from Figure 4, the accuracy of the force approximation should vary from step to step. The size of these fluctuations is measured by the standard deviation, $\sigma_{\eta}=\left\langle(\eta-\langle\eta\rangle)^{2}\right\rangle^{1 / 2}$.

Taking the point of view of numerical mathematics one might expect that $\langle\eta\rangle$ and $\sigma_{\eta}$ represent measures for the algorithmic noise associated with a given approximation method. However, as discussed in Refs. 16 and 20, there are physical considerations contradicting that expectation. For instance, the DC-1d extrapolation scheme has been designed to guarantee optimal energy conservation and other useful properties. Correspondingly, that method has been demonstrated to entail smaller algorithmic noise in the framework of pure force extrapolation than the linear extrapolation scheme, although it exhibits larger values of $\langle\eta\rangle$ and $\sigma_{\eta}$ than the latter. ${ }^{16,20}$ It remains to be seen whether this is also the case in our framework of local Taylor expansion extrapolations. Therefore, we also have to consider the size of uncontrolled algorithmic noise. In the microcanonical simulations at hand the estimation of that size is trivial. As algorithmic noise is the sole cause for energy transfer into such systems, one simply has to monitor the drift of the total energy, $\Delta E_{\text {total }} / \Delta T$.

\section{LONG TIME OBSERVABLES}

To estimate the influence of the applied approximations on the description of protein structure and dynamics, we have extracted the following observables from the long time simulations. As a measure for the temporal evolution of a protein structure we have chosen the rms deviations $\rho_{\alpha}(t)$ 
of the intermediate configurations from the initial structure. If $\mathbf{r}_{i}(0)$ are the atomic positions obtained after the equilibration phase, the rms deviations of the set, $I_{B}$, of heavy backbone atoms $(\alpha=B)$ and of the corresponding set, $I_{S}$, of side chain atoms $(\alpha=S)$ are given by:

$$
\rho_{\alpha}(t)=\left[\frac{1}{N_{\alpha}} \sum_{i \in I_{\alpha}}\left|\mathbf{r}_{i}(t)-\mathbf{r}_{i}(0)\right|^{2}\right]^{1 / 2}
$$

where $N_{\alpha}$ denotes the number of atoms in the set $I_{\alpha}$.

To estimate approximation effects on protein dynamics we monitor correlations between the motions of atoms $i$ and $j$ by the normalized covariance matrix:

$$
K_{i j}=\frac{\left\langle\left(\mathbf{r}_{\mathbf{i}}-\left\langle\mathbf{r}_{\mathbf{i}}\right\rangle\right) \cdot\left(\mathbf{r}_{\mathbf{j}}-\left\langle\mathbf{r}_{\mathbf{j}}\right\rangle\right)\right\rangle}{\left[\left\langle\left(\mathbf{r}_{\mathbf{i}}-\left\langle\mathbf{r}_{\mathbf{i}}\right\rangle\right)^{2}\right\rangle\left\langle\left(\mathbf{r}_{\mathbf{j}}-\left\langle\mathbf{r}_{\mathbf{j}}\right\rangle\right)^{2}\right\rangle\right]^{1 / 2}}
$$

where the averages are taken over trajectory sections specified further below.

Subsequently, we compare covariance matrices $K_{i j}^{A}$ and $K_{i j}^{B}$ from simulations $A$ and $B$, respectively, both by a graphical method and by a single numerical observable. The latter is the rms difference of the cross-correlations; that is:

$$
\kappa=\left[\frac{\sum_{i<j}\left(K_{i j}^{B}-K_{i j}^{A}\right)^{2}}{\sum_{i<j}\left(K_{i j}^{A}\right)^{2}}\right]^{1 / 2}
$$

whereas the former is an overlay of certain contour plots pertaining to the $K_{i j}$. These contour plots visualize histograms representing the frequencies of values $K_{i j} \in[-1,1]$ as functions of atomic distances $r_{i j}$ and will be called covariance plots.

As a technical point, we note that comparisons of the type indicated above require that the trajectories refer to one and the same conformational substate of the protein considered. The reason is that dynamical correlations may vary from substate to substate, such that differences of these correlations which are caused by the application of different algorithms may be obscured.

\section{Results of Quality Assessment}

In this section, we present the results of our study on the algorithmic accuracy of the FAMUSAMM method. First, we provide values for the short time observables extracted from the associated test simulations on the large BPTI-water system. Subsequently, we present values for the long time observables obtained for the simulations on BPTI in vacuo.

\section{SHORT TIME OBSERVABLES}

Table I compares the accuracy of force calculations achieved by the approximation schemes SAMM, FAMUSAMM/DC-1d, and FAMUSAMM/linear, respectively. As accuracy measures, Table I displays the time averages, $\langle\eta\rangle$, and standard deviations, $\sigma_{\eta}$, of the rms errors, $\eta(t)$, given by eq. (7). These values have been extracted from trajectories of 0.4-ps duration. In addition, the values for the average energy drifts, $\Delta E_{\text {total }} / \Delta T$, as calculated from trajectories of $\Delta T=60$-ps duration, are given.

As can be seen, the average rms errors $\langle\eta\rangle$ of force approximation are of about the same size (1\%) for all three methods. The $\langle\eta\rangle$ values of the FAMUSAMM algorithms are only slightly larger than that of SAMM. Thus, the multiple-time-step extrapolation procedures do not seem to sizably reduce the quality of force approximation achieved by SAMM. In contrast, as shown in Ref. 19, cutoff methods exhibit errors $\langle\eta\rangle$ that are larger by at least a factor of 10 . Thus, as far as the quality of force approximation is concerned, the FAMUSAMM procedures essentially preserve the advantageous properties of SAMM.

But, considering the fluctuations $\sigma_{\eta}$ of the error $\eta(t)$, a distinct difference between SAMM and the FAMUSAMM schemes becomes apparent. Due to the application of the multiple-time-step extrapolation procedures, for FAMUSAMM the fluctuations $\sigma_{\eta}$ are tenfold larger than for SAMM. Thus, the

\section{TABLE I.}

\section{Approximation Errors of Force Calculation for Various Methods As Measured by Time Average $\langle\eta\rangle$ of rms Error [Eq. (7)] and by Its Standard Deviation $\left(\sigma_{\eta}\right)$. Also Given Are Average Energy Drifts $\left(\Delta E_{\text {total }} / \Delta T\right)$ Reflecting Algorithmic Noise.}

\begin{tabular}{lccc}
\hline Method & $\langle\eta\rangle$ & $\sigma_{\eta}$ & $\begin{array}{c}\Delta E_{\text {total }} / \Delta T \\
{[\mathrm{kcal} /(\mathrm{mol} \mathrm{ps})]}\end{array}$ \\
\hline $\begin{array}{l}\text { SAMM } \\
\begin{array}{l}\text { FAMUSAMM / } \\
\quad \text { DC-1d }\end{array}\end{array}$ & $0.70 \%$ & $0.01 \%$ & 4.8 \\
$\begin{array}{l}\text { FAMUSAMM / } \\
\text { linear }\end{array}$ & $1.04 \%$ & $0.14 \%$ & 9.2 \\
\hline
\end{tabular}


question arises as to whether the slightly larger values of $\langle\eta\rangle$ and the drastically increased values of $\sigma_{\eta}$ for the FAMUSAMM methods are accompanied by comparable increases of algorithmic noise.

Consideration of the associated values for the energy drifts in Table I reveals a slight increase for FAMUSAMM/DC-1d as compared to SAMM, which is in line with the corresponding increase of $\langle\eta\rangle$. In contrast, a dramatically enhanced algorithmic noise is apparent for the FAMUSAMM/linear approach, although in that case $\langle\eta\rangle$ is nearly as small as for SAMM. Hence, the superiority of the DC-1d scheme, as compared to the linear extrapolation, is actually preserved despite the fact that, in FAMUSAMM, extrapolations are applied to local Taylor expansion coefficients representing approximated forces instead of explicitly calculated exact forces. Finally, we note that the $\sigma_{\eta}$ values are not correlated with the observed algorithmic noise; this finding underlines the validity of the respective arguments presented in Refs. 16 and 20.

\section{LONG TIME OBSERVABLES}

To investigate the influence of approximation schemes on the description of protein structure and dynamics we first analyze the temporal evolution of the rms deviations $\rho_{\alpha}(t)$ defined by Eq. (8). The graphs shown in Figure 5 have been obtained from the various 1.2-ns simulations of BPTI in vacuo. The graphs represent smoothened versions of $\rho_{\alpha}(t)$ obtained by taking local averages of 5-ps width.

The rms deviations, $\rho_{S}(t)$, of the side-chain atoms are always larger than the rms deviations, $\rho_{B}(t)$, of the backbone atoms, because side-chains are much more flexible than the protein backbone. As can also be seen in Figure 5, both $\rho_{B}(t)$ and $\rho_{S}(t)$ increase with simulation time and generally exhibit a series of jumps. The first jump reflects the thermal fluctuations around the initial structure, whereas later jumps, as shown by a closer inspection of the associated structures (data not shown), arise from conformational transitions.

Furthermore, one recognizes from the top two plots in Figure 5 that the time development of the rms deviations $\rho_{\alpha}(t)$ distinctly differs for the two reference simulations, indicating that, indeed, the chaotic character of the system has led to a complete decorrelation of the respective trajectories. Yet the rms deviations resulting after $1.2 \mathrm{~ns}$ are of similar size and measure $\rho_{B} \approx 2 \AA$ and $\rho_{S} \approx 3 \AA$, respectively. Within the first 200 ps our rms deviations agree well with those obtained from other
BPTI simulations in vacuo of up to $210 \mathrm{ps}$ in length. ${ }^{2,6}$

Inspection of the subsequent three plots in Figure 5 shows that, for SAMM and FAMUSAMM, the overall behavior of $\rho_{\alpha}(t)$ is similar to that obtained for the two reference simulations. All graphs exhibit jumps pointing toward conformational transitions, and after 1.2 ns the values of $\rho_{\alpha}(t)$ are close to those of the reference simulations.

In contrast, $\rho_{\alpha}(t)$ exhibits a qualitatively and quantitatively different behavior for the cutoff simulation (lower right plot; note the different scale). Here, one single, large conformational transition occurs after about 100 ps, and subsequently conformational transitions are suppressed." As shown by an analysis of the protein structure, the transition was connected with a contraction upon which its overall size was reduced by about $5 \AA$.

In summary, as monitored by $\rho_{\alpha}(t)$, no essential differences can be detected between the reference simulations, SAMM, and FAMUSAMM simulations, whereas the cutoff simulation apparently describes a different system.

We now turn to the investigation of dynamical correlations as described by the covariance matrices $K_{i j}$ defined in eq. (9). As noted previously, a comparison of such matrices requires that the trajectories, from which the $K_{i j}$ values are calculated, refer to identical or at least similar conformational substates of the protein. Inspection of Figure 5 shows that one can identify a couple of sections within the trajectories of the simulations pointing to conformational substates that are stable for at least 200 ps. These sections marked by capital letters in Figure 5 were employed for evaluation of associated covariance matrices.

Differences between the covariance matrices calculated in this way can be due to various sources: (i) they may represent pure statistical fluctuations; (ii) they may reflect dynamical variations associated with the individual conformational substates; and (iii) they may be caused by the application of deviating computational methods.

To disentangle these sources we first provide an estimate for (i) the size of statistical fluctuations. For this purpose we chose the two consecutive 200-ps sections of the second reference trajectory, which are marked in Figure 5 (top right, A and B). As is indicated by the constancy of the $\rho_{\alpha}$ in that period, these sections refer to one and the same

"To assure this observation, we have performed a second cutoff simulation and have observed similar effects. 


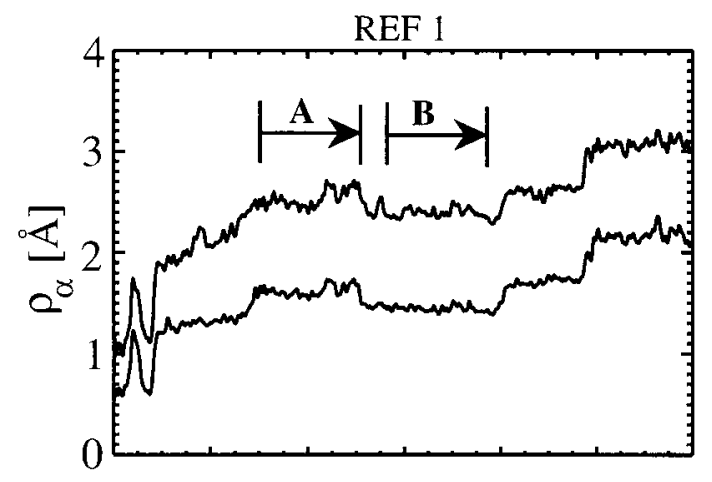

REF 2
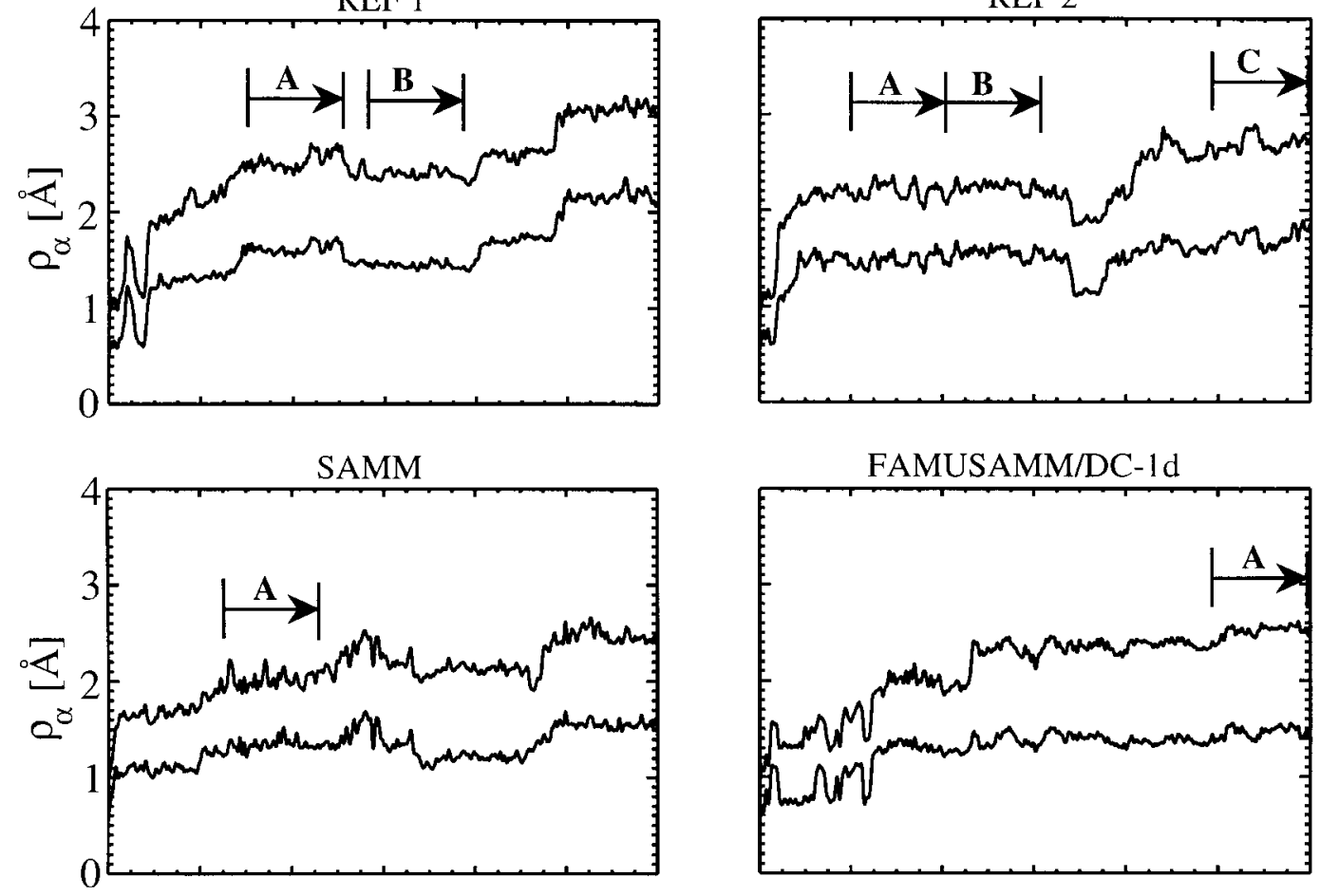

FAMUSAMM/DC- $1 \mathrm{~d}$
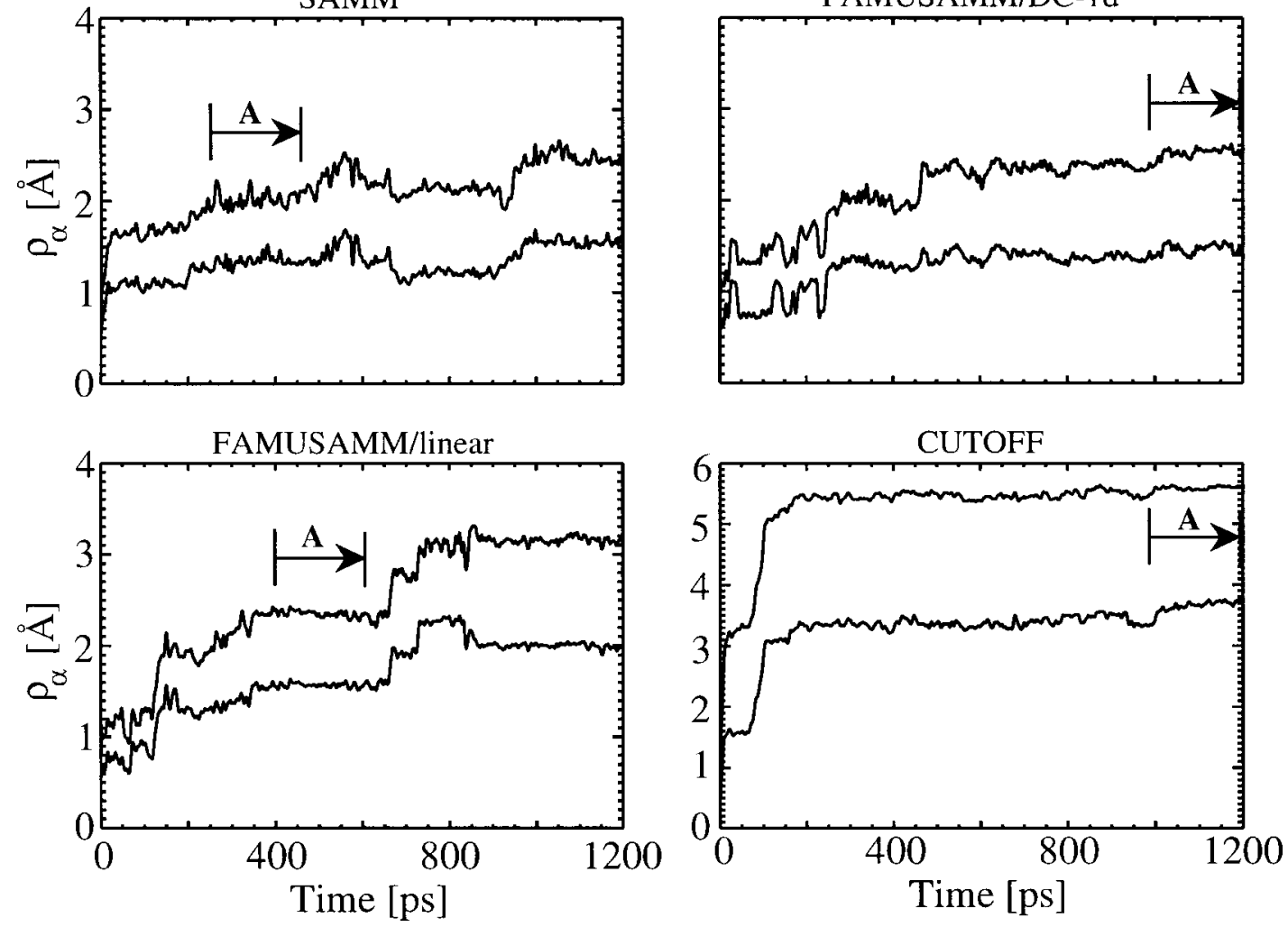

FIGURE 5. Temporal evolution of backbone rms deviations, $\rho_{B}$, and side-chain rms deviations, $\rho_{S}$, for the two reference simulations (REF1 and REF2) and the four approximation methods. Due to the flexibility of the side chains, $\rho_{S}$ is always larger than $\rho_{B}$; trajectory sections, which correspond to stable substates and are used for later evaluation of the covariance matrices, are denoted by capital letters. For explanation see text.

conformational substate of BPTI. Figure 6a shows an overlay of the two covariance plots calculated from the covariance matrices associated with these trajectory sections and displays the value of the rms difference $\kappa$ between the covariance matrices.

As one can see, the two plots are nearly identical. They show strong positive correlations between motions of neighboring atoms, which reflect the rigidity of the covalently bonded structure. At intermediate distances around $12 \AA$, anticorrelations dominate, whereas correlations be- come positive again at distances larger than $25 \AA$. The rms deviation, $\kappa$, of the two cross-correlations is $30 \%$. Thus, deviations $\kappa$ have to be distinctly larger than that value, and covariance plots have to exhibit much more pronounced differences than those in Figure 6a, to exclude mere effects of statistical fluctuations and to prove the existence of physical or algorithmic differences of the simulated dynamics.

A measure for the alteration of correlations of atomic motion, which is induced by a conforma- 

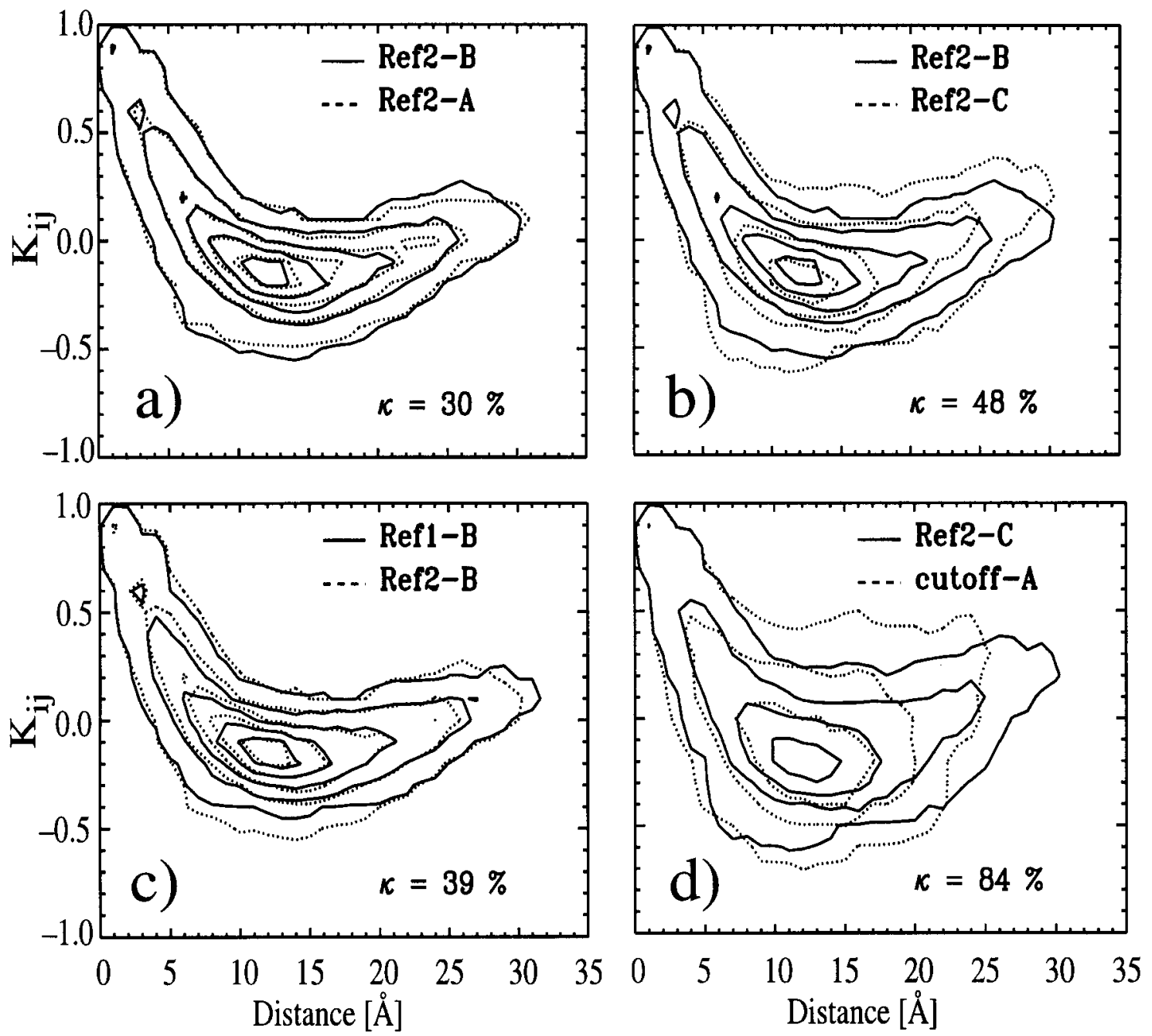

FIGURE 6. Covariance plots for comparison of BPTI dynamics within stable conformational substates simulated by different methods (see text for explanation).

tional transition into a different substate [see point (ii)], is provided by Figure $6 \mathrm{~b}$. This figure also shows covariance matrices extracted from the second reference trajectory, but, in contrast to Figure $6 a$, trajectory sections referring to different substates of BPTI are compared. Specifically, sections $B$ and $C$, as marked in Figure 5 (top right), have been chosen for comparison. In comparison with Figure 6a, the covariance plots exhibit sizable differences, and the rms deviation of cross-correlations, measuring $\kappa=48 \%$, is larger. But note that, apparently, the conformational transition from state $B$ to state $C$ has had little influence on the overall shape of the covariance plots.

Similar conclusions may be drawn if one considers Figure $6 c$. This figure compares covariance plots extracted from different trajectories calculated with the same computational method; that is, sections $B$ of the first reference simulation and section B of the second reference simulation as marked in Figure 5 (top plots). These sections refer to slightly different substates, which have been selected according to a criterion of maximal structural similarity. Consequently, the good match of the covariance plots in Figure $6 c$, as well as the low value of $\kappa=39 \%$, indicates that closely resembling structures exhibit similar dynamics.

To finally pin down the range within which the rms deviations $\kappa$ of cross-correlations can vary upon conformational transitions at a nanosecond time scale, we have additionally selected a whole set of 200-ps sections corresponding to stable sub- 

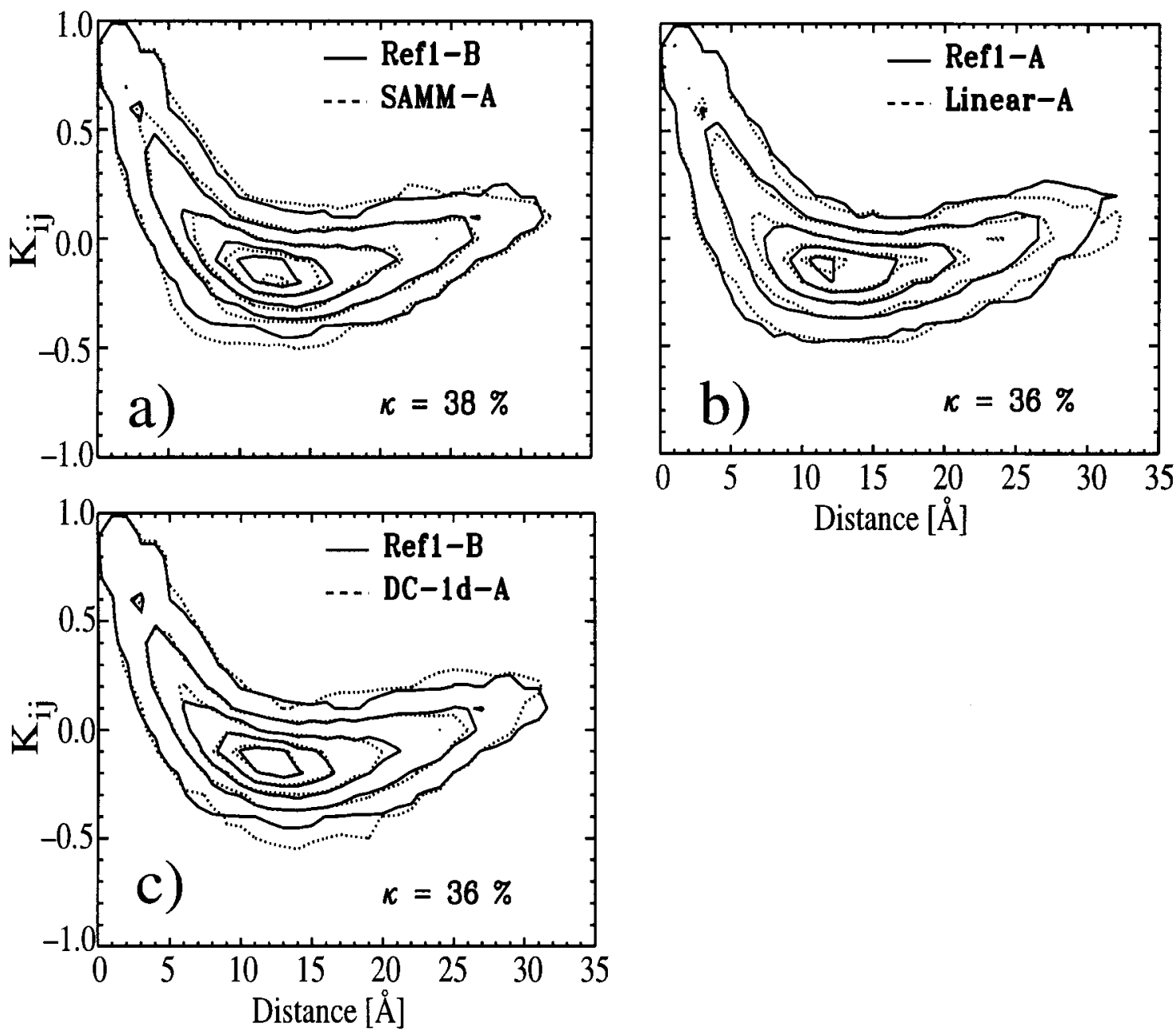

Distance $[\AA]$

FIGURE 7. Further covariance plots for evaluation of our simulation methods.

states from the two reference trajectories. We found that $\kappa$ never exceeded $70 \%$ and had a mean value of $58 \%$.

We now are in a position to use our long time observables to distinguish algorithmic artifacts from effects of statistical fluctuations and conformational transitions. For this purpose we will select from the trajectories calculated with the approximate methods 200 ps sections with the following properties: (a) they should refer to stable substates; and (b) the structure of that substate should be as similar as possible to the structure of one of the substates sampled in one of the reference trajectories.

Figure $6 \mathrm{~d}$ clearly indicates that the cutoff method is prone to algorithmic artifacts concerning the description of dynamics. This conclusion is validated, in particular, by the large value of $84 \%$ for the rms deviation, $\kappa$, which, in contrast to the covariance plot, solely expresses dynamical properties; note that the contour plots additionally display distance information.

Quite in contrast to the cutoff method all approximate methods, which account for long range Coulomb interactions by structure-adapted multipole expansions, show excellent performance in our test on dynamics. This is proven in Figure 7, which displays the respective covariance plots and the values of $\kappa$. As monitored by the plots and by $\kappa$, in all cases, the substate dynamics calculated by SAMM and FAMUSAMM resembles quite closely the dynamics of a similar substate sampled by the reference simulations. The comparisons are as close as those between sections $B$ of the reference simulations shown in Figure 6c. Furthermore, we have also selected a set of 200-ps sections referring to arbitrary substates from the SAMM and FAMUSAMM trajectories for comparison with sub- 


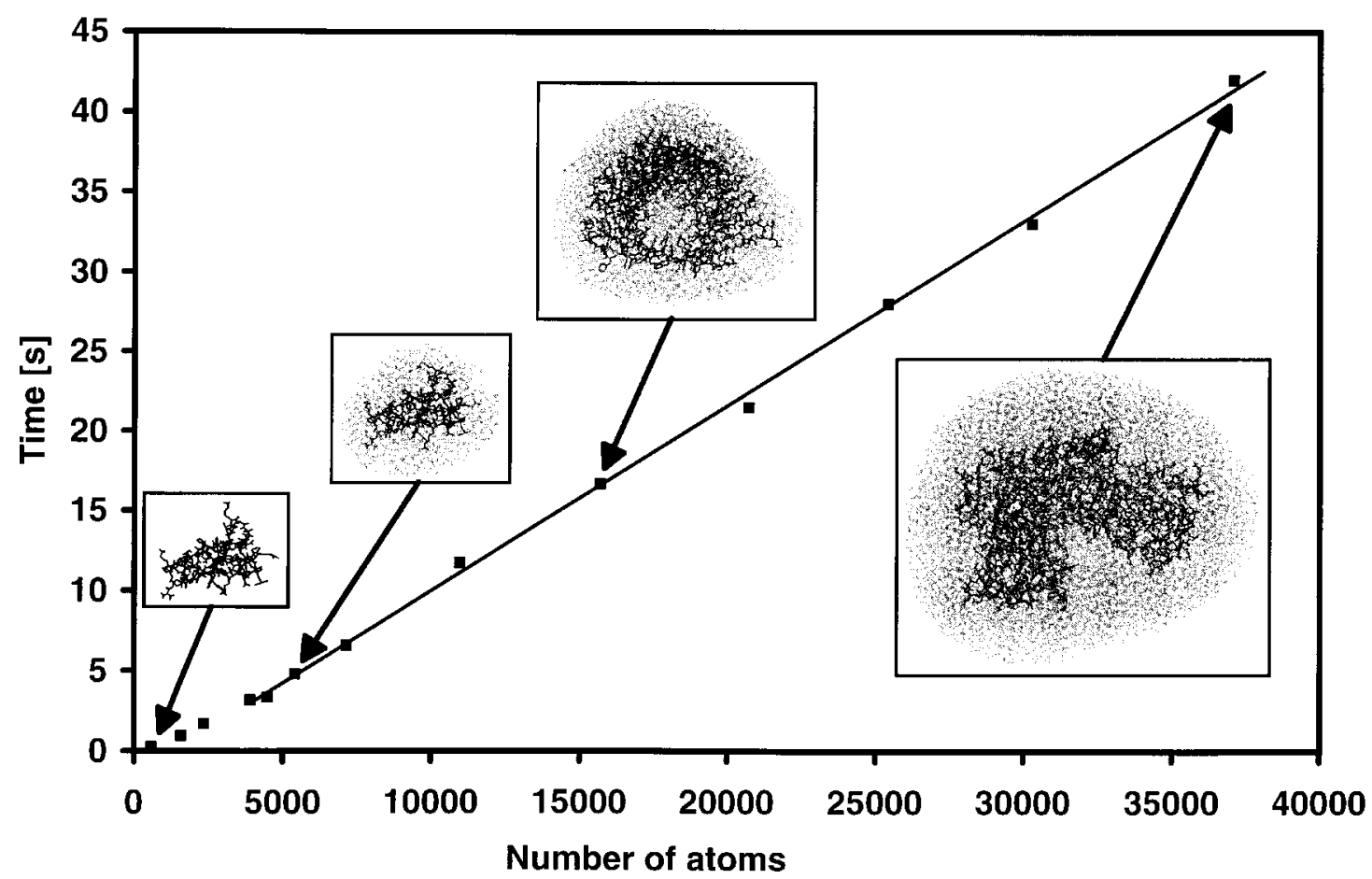

FIGURE 8. Average computation time for one step using EGO_VIII on a DEC-Alpha $3300 \mathrm{~L}$ workstation (175 MHz) for simulation systems of varying size.

state sections from both reference simulations using $\kappa$ as the observable. Like in the comparison among the reference sections noted further above, no value larger than $70 \%$ was found for $\kappa$. As a consequence, concerning dynamics, no difference has been detected between the exact evaluation of long range Coulomb forces and our approximate methods.

Summarizing the results of our study on the accuracy of the various approximate MD algorithms we can state that the FAMUSAMM/DC-1d algorithm: (a) provides forces that are nearly as accurate as those obtained with SAMM; (b) entails comparably little algorithmic noise; and (c) also passes our tests on the description of protein structure and dynamics with equally excellent results. In contrast, FAMUSAMM/linear is hampered by pronouncedly increased algorithmic noise, whereas the cutoff method exhibits unsatisfactory behavior in every respect. In view of these results we have discarded the linear multiple-time-step extrapolation scheme from further consideration within FAMUSAMM. It remains to be seen the extent to which the favorable algorithmic properties of FAMUSAMM are accompanied by a correspondingly favorable computational performance.

\section{COMPUTATIONAL PERFORMANCE}

Here we show that FAMUSAMM actually provides an enhanced computational efficiency both as compared to SAMM as well as to the reference method with its exact evaluation of the Coulomb sum. For this purpose, we first consider how the computational effort of FAMUSAMM scales with system size.

For a quantitative performance assessment we have carried out a series of test simulations on systems of varying size using the sequential version of our MD program EGO_VIII. The chosen system sizes covered the range from 500 to 40,000 atoms. All simulations were executed on a DECALPHA 3300L (175 MHz) workstation equipped with 96 MB RAM (due to limitations of memory we were unable to extend our tests to larger systems). Figure 8 shows how the average computation time required for one MD integration step scales with system size. The choice of an average computation time for performance measurements is necessary, because, in FAMUSAMM, the time required for the force calculation varies from time step to time step (cf. Fig. 4).

Figure 8 clearly shows that, for systems comprising more than about 1000 atoms, FAMUSAMM 
achieves linear scaling of computational effort with size. The quadratic increase observed for very small systems is in line with the fact that, in the innermost distance class ( $H=0$ in Fig. 4), forces are calculated by use of the Coulomb sum with its intrinsically quadratic scaling behavior. Thus, the theoretically expected linear scaling of FAMUSAMM for large systems is actually obtained in our implementation.

For performance comparisons with other methods consideration of system size is essential. Compared with its parent method, SAMM, our new algorithm achieves a speed-up by a factor of two for small systems containing less than about 5000 atoms. This gain of efficiency is entirely due to our multiple-time-step extrapolation of forces in the innermost SAMM distance class $H=0$ (see subsection "Inner Class Force Extrapolation" in section Combination of SAMM with a MultipleTime-Step Method, as well as Fig. 4). Note that SAMM had been previously demonstrated to perform for systems up to that size as efficiently as the conventional cutoff methods. ${ }^{26}$ Thus, FAMUSAMM is more favorable than the cutoff method in regard to efficiency.

For large systems comprising 36,000 atoms FAMUSAMM performs four times faster than SAMM and as fast as cutoff. Here, the speed-up with respect to SAMM is essentially achieved by the multiple-time-step extrapolation of local Taylor expansions in the outer distance classes, and FAMUSAMM executes 60 times faster than the reference method based on the evaluation of the Coulomb sum.

\section{Summary and Conclusion}

In this study we have combined the fast SAMM method for approximate evaluation of long range Coulomb interactions in MD simulations of protein dynamics with the DC-1d procedure designed for the same purpose into a new and highly efficient MD method. Upon extended test simulations the resulting FAMUSAMM algorithm has been demonstrated to preserve and combine the favorable properties of its parent methods concerning accuracy, lack of sizable algorithmic artifacts, and last, but not least, computational efficiency. Thus, FAMUSAMM opens the way for extended MD simulations which properly account for the long range Coulomb interactions of large proteinsolvent systems comprising several tens of thousands of atoms.
The algorithm has been implemented in the MD program EGO_VIII, which is based on the CHARMM force field, in a sequential and parallelized version, and is available via the internet. ${ }^{51}$ Whereas the current study is exclusively devoted to the presentation and evaluation of our method, a previously published application ${ }^{62}$ has already demonstrated its suitability for the quantitative analysis of experimental findings; there, the method has enabled an atomic interpretation of atomic force microscopy experiments on ligandreceptor binding. ${ }^{63}$ As a verification of the FAMUSAMM MD simulation performed in Ref. 62, the rms deviation from the x-ray structure of a solvated protein (streptavidin in water, 10,969 atoms) has been monitored. After 0.5-ns simulation, an rms deviation as small as $1.5 \AA$ was found, showing that, indeed, FAMUSAMM provides realistic descriptions of solvated proteins.

\section{Appendix: Pseudo-Code of FAMUSAMM}

In this section we provide a summarizing pseudo-code description of FAMUSAMM. For clarity, we have omitted the special algorithmic steps, which are necessary during the startup phase or are associated with hierarchical grouping and recalculation of the interaction list. To decide whether, in a given time step, forces or local Taylor expansions have to be explicitly calculated or may be extrapolated, we introduce a variable, $M$, which denotes the highest hierarchy level, $H$, of SAMM for which an explicit calculation has to be performed (cf. Fig. 4). $M$ is called the extrapolation level. The following abbreviations are used: LTE: local Taylor expansion; MM: multipole moments; and SU: structural unit. Indentation defines the grouping of instructions.

For every integration step:

Set extrapolation level $M$.

Set all LTE coefficients to zero.

Calculate MMs of all SUs (hierarchy level $H=1$ ).

For $H=2$ to $H=H_{\max }$ :

Calculate MMs for each cluster on higher hierarchy levels $H$.

For $H=H_{\max }$ down to $H=1$ :

For every object at level $H$ :

if $H>M$ :

Extrapolate LTE of hierarchy level $H$. Else:

Sum up contributions to LTE at level 
$H$ according to interaction list

If $H>1$ :

Inherit LTE of level $H$ to level $H-1$.

\section{For every atom:}

Compute nonelectrostatic forces forces (van der Waals, chem. binding forces).

Add Coulomb forces from LTE of SU.

Add Coulomb forces from atoms in distance class $j=0$ (cf. Fig. 4).

If integration step even:

Add calculated forces from distance class

$j=1$.

class $j=1$.

Else:

Add extrapolated forces from distance

Perform Verlet integration step.

\section{References}

1. M. Levitt and S. Lifson, J. Mol. Biol. 46, 269 (1969).

2. J. A. McCammon, B. R. Gelin, and M. Karplus, Nature (London), 267, 585 (1977).

3. W. F. van Gunsteren and H. J. C. Berendsen, Mol. Phys., 34, 1311 (1977)

4. O. Edholm, O. Berger, and F. Jähnig, J. Mol. Biol., 250, 94 (1995).

5. H. Heller, M. Schaefer, and K. Schulten, J. Phys. Chem. 97, 8343 (1993).

6. M. Levitt and R. Sharon, Proc. Natl. Acad. Sci. USA, 85, 7557 (1988).

7. M. Levitt, Chem. Scr., 29A, 197 (1989).

8. M. C. Nuss, W. Zinth, W. Kaiser, E. Kölling, and D. Oesterhelt, Chem. Phys. Lett., 117, 1 (1985).

9. G. Petsko, Nature (London), 371, 740 (1994).

10. F. Zhou, A. Windemuth, and K. Schulten, Biochemistry, 32, 2291 (1993).

11. M. P. Allen and D. Tildesley, Computer Simulations of Liquids, Clarendon Press, Oxford, 1987.

12. J. A. McCammon and S. C. Harvey, Dynamics of Proteins and Nucleic Acids, Cambridge University Press, Cambridge, 1987.

13. W. F. van Gunsteren and H. J. C. Berendsen, Angew. Chem. Int. Ed. Engl., 29, 992 (1990).

14. B. R. Brooks, R. E. Bruccoleri, B. D. Olafson, D. J. States, S. Swaminathan, and M. Karplus, J. Comput. Chem., 4, 187 (1983).

15. C. L. Brooks III, B. M. Pettit, and M. Karplus, J. Chem. Phys., 83, 5897 (1985).

16. H. Grubmüller, Molekulardynamik von Proteinen auf langen Zeitskalen, Dissertation, Technische Universität München, Germany, 1994.

17. R. J. Loncharich and B. R. Brooks, Proteins, 6, 32 (1989).

18. C. Niedermeier, Modellierung elektrostatischer Wechselwirkungen in Proteinen: Eine strukturadaptierte Multipolmethode, Dissertation, Ludwig-Maximilians-Universität, München, Germany (1995).
19. C. Niedermeier and P. Tavan, J. Chem. Phys., 101, 734 (1994).

20. H. Grubmüller and P. Tavan (in press).

21. A. W. Appel, SIAM J. Sci. Stat. Comput., 6, 85 (1985).

22. J. Barnes and P. Hut, Nature (London), 324, 446 (1986).

23. L. Greengard and V. Rokhlin, J. Comput. Phys., 73, 325 (1987).

24. L. Greengard and V. Rokhlin, Chem. Scr., 29A, 139 (1989).

25. J. F. Leathrum and J. A. Board, The Parallel Fast Multipole Algorithm in Three Dimensions, Technical Report, Department of Electrical Engineering, Duke University, Durham, NC, 1992.

26. C. Niedermeier and P. Tavan, Mol. Sim., 17, 57 (1996).

27. A. Ahmad and L. Cohen, J. Comput. Phys., 12, 389 (1973).

28. W. B. Streett, D. J. Tildesley, and G. Saville, Mol. Phys., 35, 639 (1978).

29. R. C. Y. Chin, G. W. Hedstrom, and F. A. Howes, Considerations on Solving Problems with Multiple Scales, Academic Press, Orlando, FL, 1985.

30. M. E. Tuckerman, G. J. Martyna, and B. J. Berne, J. Chem. Phys., 93, 1287 (1990).

31. M. E. Tuckerman, B. J. Berne, and G. J. Martyna, J. Chem. Phys., 94, 6811 (1991).

32. H. Grubmüller, H. Heller, A. Windemuth, and K. Schulten, Mol. Sim., 6, 121 (1991).

33. R. D. Skeel and J. J. Biesiadecki, Ann. Num. Math., 1, 191 (1994).

34. J. A. Board, Jr., J. W. Causey, J. F. Leathrum, Jr., A. Windemuth, and K. Schulten, Chem. Phys. Lett., 198, 89 (1992).

35. H.-Q. Ding, N. Karasawa, and W. A. Goddard III, J. Chem. Phys., 97, 4309 (1992).

36. M. O. Fenley, W. K. Olson, K. Chua, and A. H. Boschitsch, J. Comput. Chem., 17, 976 (1996).

37. A. Windemuth, In T. G. Mattson, Ed., Parallel Computing in Computational Chemistry, ACS Books, Washington, DC, 1995.

38. R. Zhou and B. J. Berne, J. Phys. Chem., 103, 9444 (1995).

39. P. P. Ewald, Ann. Phys. (Leipzig), IV, 253 (1920).

40. A. Y. Toukmaji and J. J. A. Board, Comput. Phys. Commun., 95, 73 (1996).

41. E. L. Pollock and J. Glosli, Comput. Phys. Commun., 95, 93 (1996).

42. U. Essmann, L. Perera, M. L. Berkowitz, T. Darden, H. Lee, and L. G. Pedersen, J. Chem. Phys., 103, 8577 (1995).

43. P. Procacci, T. Darden, and M. Marchi, J. Phys. Chem., 100, 10464 (1996).

44. C. L. Brooks III and M. Karplus, J. Chem. Phys., 79, 6312 (1983).

45. D. Beglov and B. Roux, J. Chem. Phys., 100, 9050 (1994).

46. P. E. Smith and B. M. Pettit, J. Chem. Phys., 105, 4289 (1996).

47. H. Bekker, Molecular Dynamics Simulation Methods Revised, Dissertation, Rijksuniversiteit Groningen, Groningen, The Netherlands, 1996.

48. A. Brünger, C. Brooks, and M. Karplus, Chem. Phys. Lett., 105, 495 (1994).

49. T. M. Martinetz and K. J. Schulten, In Proceedings of the International Conference on Artificial Neural Networks, ICANN-91, Espoo, Finland, 24-28 June 1991, O. Simula, Ed., Elsevier, Amsterdam, 1991, p. 397. 
50. M. Eichinger, Paralleler schneller Multipolalgorithmus mit Mehrschrittverfahren für Molekulardynamiksimulationen, Diplomarbeit, Ludwig-Maximilians-Universität, München, Germany, 1995.

51. M. Eichinger, H. Grubmüller, and H. Heller, User Manual for EGO_VIII, Release 2.0, Theoretische Biophysik, Institut für Medizinische Optik, Ludwig-Maximilians-Universität, Theresienstr. 37, D-80333 München, Germany, 1995. Electronic access: http://www.imo.physik.uni-muenchen.de/ ego.html.

52. M. Eichinger, H. Grubmüller, and H. Heller, in preparation.

53. S. Hayward, A. Kitao, and N. Gō, Physica Scripta, 3, 936 (1994).

54. S. Swaminathan, T. Ichiye, W. van Gunsteren, and M. Karplus, Biochemistry, 21, 5230 (1982).

55. M. Saito, Mol. Sim., 8, 321 (1992).
56. W. L. Jorgensen, J. Chandrasekhar, and J. D. Madura, J. Chem. Phys., 79, 926 (1983).

57. A. Brünger, X-PLOR Manual, The Howard Hughes Medical Institute and Department of Molecular Biophysics and Biochemistry, Yale University, New Haven, CT, 1992.

58. C. Eckart, Phys. Rev., 47, 552 (1935).

59. N. Ehrenhofer, Untersuchung der Konformationsdynamik eines vereinfachten Proteinmodells, Diplomarbeit, Ludwig-Maximilians-Universität, München, Germany, 1994.

60. H. C. Anderson, J. Chem. Phys., 72, 2384 (1980).

61. H. J. C. Berendsen, J. P. M. Postma, W. F. van Gunsteren, A. DiNola, and J. R. Haak, J. Chem. Phys., 81, 3684 (1984).

62. H. Grubmüller, B. Heymann, and P. Tavan, Science, 271, 997 (1996).

63. E.-L. Florin, V. T. Moy, and H. E. Gaub, Science, 264, 415 (1994). 